\title{
Long term complications of grommet insertion in paediatric patients
}

\author{
N. Titus, N. Vakharia, A. Jafri, A. Salem, Y. Bajaj \\ Royal London Hospital, Barts Health NHS Trust, London \\ Corresponding author: Nilesh.Vakharia@nhs.net
}

\section{INTRODUCTION}

Otitis media with effusion, (OME, 'Glue Ear') is a common condition of childhood where management via the insertion of grommets is widely implemented.

Every year in the UK over 30,000 patients undergo insertion of grommets. After tooth extraction, grommet insertion is the second most common reason a child has general anaesthetic in the UK [1].

Complications are common however, and informed consent of patients is vital. The Montgomery ruling further highlights the importance of taking reasonable care to ensure that the patient is aware of any material risks and long term complications involved in any recommended treatment.

The aim of this study therefore was to establish our long term complications rates over a three year period and compare these with established data.

\section{METHODS}

Retrospective analysis of paediatric patients aged $1-12$ presenting with unilateral or bilateral otitis media with effusion, for which they underwent grommet insertion at a tertiary centre between January 2014 \& December 2016.

Data for the study was extracted from electronic patient records, by reviewing all relevant clinic letters and discharge summaries.

\section{Fig 1. OUTCOME OF GROMMET INSERTION WITH} BREAKDOWN OF COMPLICATIONS

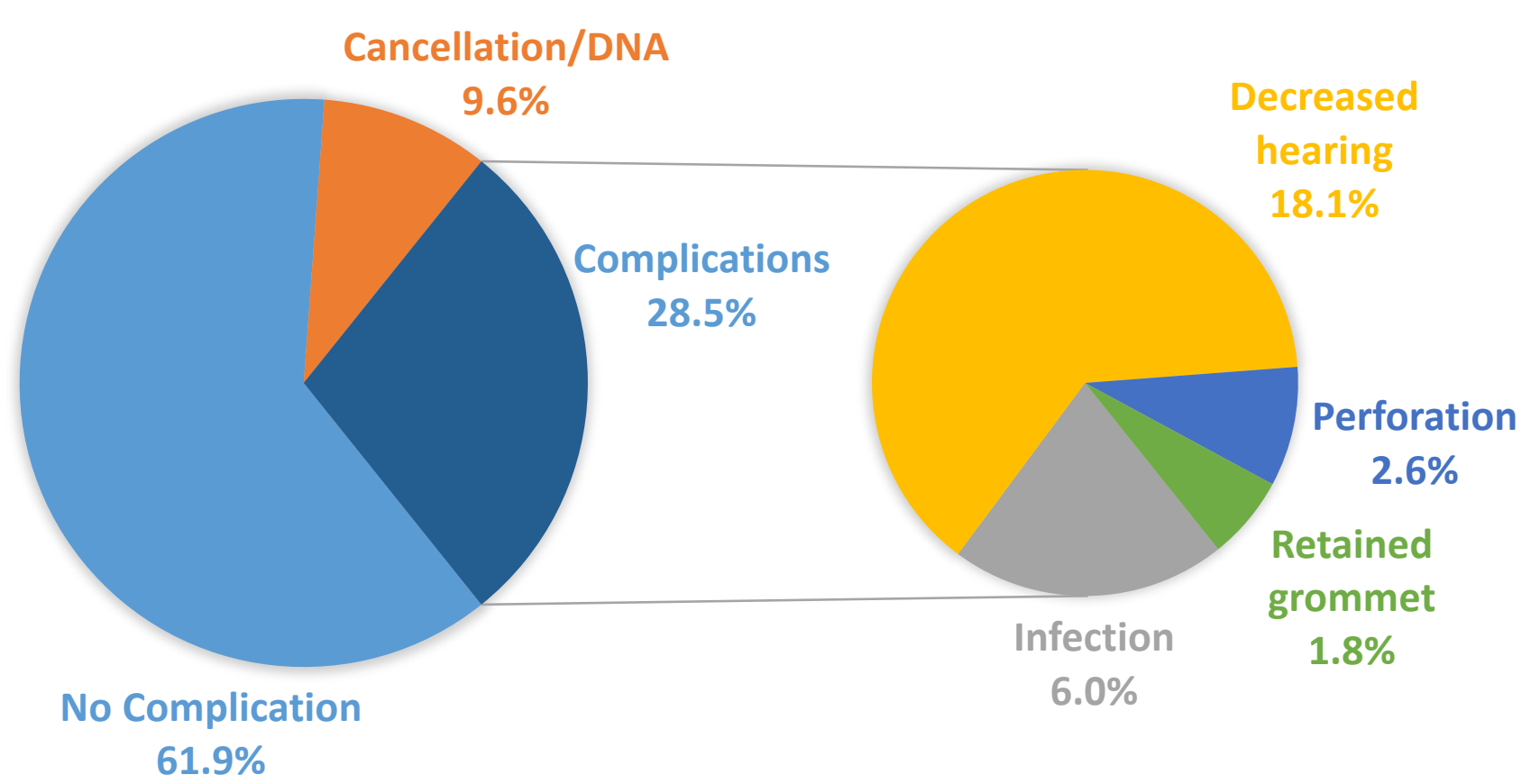

RESULTS

We included a total of 386 participants who between January 2014 and December 2016 underwent grommet insertion.

$110(28.6 \%)$ (Fig. 1 ) of these patients developed complications secondary to this procedure which was then subdivided into the following four groups:

- Decreased hearing secondary to recurrence of OME $18.1 \%$

- Infection 6\%

- Persistent perforation $2.6 \%$

- Retained grommet $1.8 \%$

At six month follow-up, 239 (61.9\%) patients had resolution of symptoms and suffered no complications secondary to grommet insertion and thus required no further input.

The complications seen developed over a period averaging 14 months after the insertion of the grommet. No association was identified between risk of complication and patient co-morbidity.

In terms of of patient outcomes (Fig.2), of the 386 patients listed for grommet insertion:

- $12.4 \%(48 / 386)$ required further surgical intervention for their management

- $4.1 \%(16 / 386)$ were placed on a waiting list for further intervention secondary to a complication

- $11.9 \%$ (46/386) were managed conservatively through measures such as antibiotics, hearing aids and Otovent.

\section{Fig 2. SUMMARY OF PATIENT OUTCOMES}

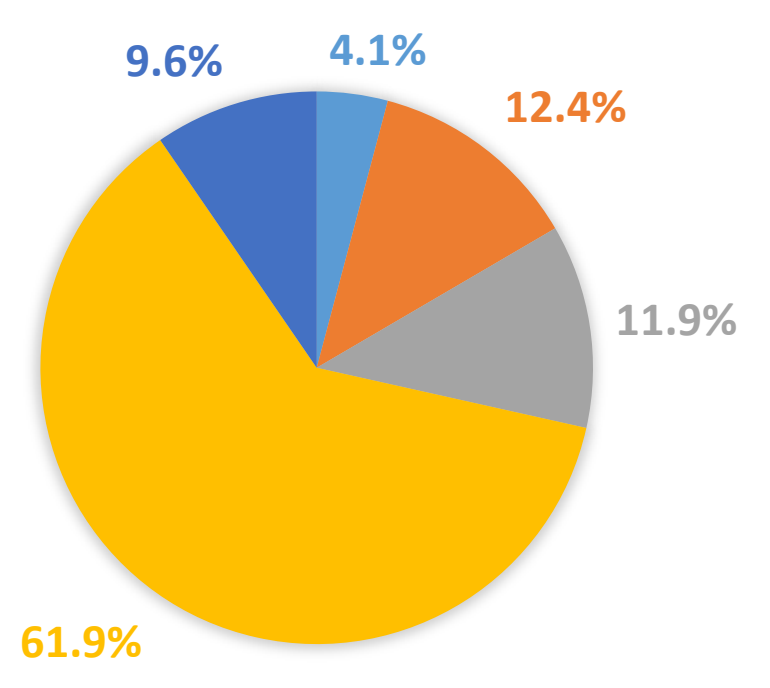

Waiting list for further intervention

- Received additional

surgical management

- Conservative

Management

- No Complications/

Discharge

- DNA/Cancellation

\section{CONCLUSION}

We found that our complication rate post grommet insertion of $28.6 \%$ was significantly lower than current literature that reports rates reaching as high as $80 \%$ in operated ears under specific circumstances and within certain subgroups [2].

It is a common pre-operative practice to convey the risk of perforation being up to $5 \%$ [1]. This is further supported by a meta-analysis of over 62 studies totalling 20,222 ears that found a chronic perforation incidence of $4.8 \%$ [3]. Our rate, however was found to be $2.6 \%$.

Current literature reports the percentage of children who require revision surgery in the two-year follow up period from grommet insertion to lie between $28-35 \%$ [4], however only $12.4 \%$ of the patients in our cohort required further surgical procedures.

When gaining consent, these reported lower complication rates will not only give parents/caregivers a more accurate post-operative picture but may also influence them to proceed with what is an extremely safe and efficacious procedure. 